\title{
A Retrospective Study of Clinicoepidemiological Aspects of Nasopharyngeal Cancer at NCI-Cairo (2000-2010)
}

\author{
Hebatallah K. Ibrahim1, Tarek Shouman1, Azza Niazy Taher'1, Azza M. Nasr ${ }^{1}$, \\ Hesham Atef ${ }^{2}$ \\ ${ }^{1}$ Radiation Oncology Department, National Cancer Institute, Cairo University, Cairo, Egypt \\ ${ }^{2}$ Clinical Oncology Department, Faculty of Medicine, Cairo University, Cairo, Egypt \\ Email: nasrazza2@hotmail.com
}

Received 3 September 2015; accepted 11 October 2015; published 14 October 2015

Copyright (C) 2015 by authors and Scientific Research Publishing Inc.

This work is licensed under the Creative Commons Attribution International License (CC BY).

http://creativecommons.org/licenses/by/4.0/

(c) (i) Open Access

\begin{abstract}
Aim of Work: The aim of this work was to study the clinico-epidemiological characteristics of the patients with nasopharyngeal carcinoma (NPC), analyse the prognostic factors of the disease and to analyse the results of different treatment modalities and their effect on loco-regional, distal metastatic disease control and both overall survival (OS) and disease free survival (DFS) rates. Patients and Methods: This is a retrospective study reviewing all adult nasopharyngeal carcinoma (NPC) patients who presented to the radiotherapy department-National Cancer Institute Cairo University in the period from (2000-2010). Results: In this study, it was found that the mean age was 45 years; most of the patients were of locally advanced stages. Multivariate cox proportional hazards regression identified T-stage, radiotherapy course completion $\&$ addition of chemotherapy as independent prognostic factors for local control (LC), DFS, \& OS. The 5-year LC, DFS and OS rates for all studied patients were $38.2 \%, 33.5 \% \& 37.2 \%$ respectively. The median DFS was 26 months and median OS was $\mathbf{3 6 . 5}$ months. Conclusion: This study matches the published data that support that radical concurrent chemoradiation is the mainstay of treatment of locally advanced NPC, \& that T-stage, M-stage, prescribed dose completion, response to initial treatment are independent prognostic factors for survival. All measures should be taken to improve the local response during primary treatment as this will improve survival rates of patients with NPC.
\end{abstract}

\section{Keywords}

Nasopharyngeal Cancer, Radiotherapy

\footnotetext{
${ }^{*}$ Corresponding author.

How to cite this paper: Ibrahim, H.K., Shouman, T., Taher, A.N., Nasr, A.M. and Atef, H. (2015) A Retrospective Study of Clinicoepidemiological Aspects of Nasopharyngeal Cancer at NCl-Cairo (2000-2010). Journal of Cancer Therapy, 6, 971-980. http://dx.doi.org/10.4236/jct.2015.611105
} 


\section{Introduction}

Worldwide, there are 80,000 incident cases of nasopharyngeal carcinoma and 50,000 deaths annually [1]. The distinctive racial/ethnic and geographic distribution of NPC worldwide suggests that both environmental factors and genetic traits contribute to its development [2]. These unique pathogenic factors are variable such as genetic susceptibility, Epstein-Barr virus (EBV) infection, chemical carcinogens, and environmental factors [3].

The tumor-node-metastasis (TNM) staging system for malignancies is used to evaluate prognosis, aid treatment planning, and facilitate the stratification of treatment. At present, the seventh edition of the American Joint Committee on Cancer (AJCC) staging system is widely used [4].

Nasopharyngeal carcinoma (NPC) commonly demonstrates extensive invasion of adjacent tissues with poorly defined and large tumors in close proximity to critical structures, such as the brain stem, spinal cord, and optic chiasm. These features of NPC can complicate adequate surgical resection [5].

Nasopharyngeal carcinoma is highly sensitive to radiotherapy or chemotherapy [6]. Radiotherapy (RT) alone or combined with chemotherapy is the primary treatment depending on the disease stage [7].

Because of the improved treatment outcome and toxicity profile, IMRT (intensity modulated radiotherapy) is now recommended for definitive treatment for all patients with nasopharyngeal cancer [8].

\section{Aim of the Work}

This study was conducted to evaluate the clinical and epidemiological characteristics of the patients with NPC who were treated at radiotherapy department, NCI, in the last 10 years. And to study patient management and its outcomes, in addition to analyze potential prognostic factors influencing local control, disease free survival as well as overall survival of the disease

\section{Patients and Methods}

This is a retrospective study of adult nasopharyngeal cancer patients who were treated at the radiotherapy department of the National cancer institute of-Cairo university, in the period between 2000 and 2010.

The study included 158 patients; all of them were with pathologically confirmed nasopharyngeal carcinoma, 18 years of age and above, and with complete data files.

The patients were divided into 5 groups according to the type of treatment they received: 1-Radical RT 2-Radical concurrent chemoradiation (CCRT) 3-CCRT + adjuvant chemotherapy (CTH) (only 2 patients) 4-Induction CTH + CCRT 5-Palliative RT (Best supportive care).

Regarding radiotherapy, all patients who were treated by radical radiotherapy were treated in supine position using isocentric technique and all of them had proper fixation by a head and neck mask. Radical radiotherapy dose to the target volume ranged from 66 to 72 Gy with a spinal cord shield after 40 Gy while palliative dose was limited to $30 \mathrm{~Gy}$ to the target volume.

\section{Statistical Analysis}

Data was analyzed using IBM SPSS advanced statistics version 20 (SPSS Inc., Chicago, IL). Survival analysis was done using Kaplan-Meier method and comparison between two survival curves was done using log-rank test. All tests were two-tailed. A p-value $<0.05$ was considered significant [9].

\section{Results}

The study included 158 patients who were properly staged (Table 1).

\subsection{Treatment Modalities}

The most frequently used treatment modality in this study was induction chemotherapy plus concurrent chemo-radiotherapy (34.1\%), followed by the treatment modality of concurrent chemo-radiotherapy (32.9\%) (Table 2).

Out of the 149 patients who received radical radiotherapy, twenty three patients $(15.4 \%)$ were treated using 3D conformal radiotherapy (3D CRT) technique, while 2D technique was used for 121patients (81.2\%) (Table 3). 
Table 1. Patient's characteristics were.

\begin{tabular}{|c|c|c|}
\hline & Range & Mean and median \\
\hline Age & 18 - 77 years & $45 \mathrm{y}$ \\
\hline Gender & Number of Patients & Percent \\
\hline Male & 111 & $70.3 \%$ \\
\hline Female & 47 & $29.7 \%$ \\
\hline Smoking & Number of patients & Percent \\
\hline Yes & 93 & $58.9 \%$ \\
\hline No & 65 & $41.1 \%$ \\
\hline Clinical presentation & Patients No & Percent \\
\hline Neck swelling & 112 & $70.9 \%$ \\
\hline Headache & 38 & $24 \%$ \\
\hline Nasal tone & 32 & $20.6 \%$ \\
\hline Nasal obstruction & 25 & $15.8 \%$ \\
\hline Epistaxis & 22 & $14 \%$ \\
\hline CN palsy & 21 & $13.3 \%$ \\
\hline Dysphagia & 5 & $3.2 \%$ \\
\hline Performance & Number of patients & Percent \\
\hline I & 122 & $77.2 \%$ \\
\hline II & 24 & $15.2 \%$ \\
\hline III & 12 & $7.6 \%$ \\
\hline Pathological types & Number of patients & Percent \\
\hline Undifferentiated carcinoma & 121 & $76.6 \%$ \\
\hline Squamous cell carcinoma & 29 & $18.3 \%$ \\
\hline Others: & 8 & $5.1 \%$ \\
\hline Anaplastic carcinoma & 2 & $1.3 \%$ \\
\hline Adenocarcinoma & 2 & $1.3 \%$ \\
\hline Adenoid Cystic carcinoma & 4 & \\
\hline Stage group & Number of patients & \\
\hline I & 6 & $3.8 \%$ \\
\hline II & 16 & $10.1 \%$ \\
\hline III & 64 & $40.5 \%$ \\
\hline IVA & 35 & $22.2 \%$ \\
\hline IVB & 28 & $17.7 \%$ \\
\hline \multirow[t]{2}{*}{ IVC } & 9 & $5.7 \%$ \\
\hline & 158 & $100 \%$ \\
\hline
\end{tabular}

The patients' performance at presentation was classified according to the ECOG PERFORMANCE STATUS (Oken et al., 1982) [10]. 
Table 2. Treatment modalities.

\begin{tabular}{ccc}
\hline Treatment & Number of patients & Percent \\
\hline Radical radiotherapy & 41 & $26 \%$ \\
CCRT + adjuvant CTH & 2 & $1.3 \%$ \\
CCRT & 52 & $32.9 \%$ \\
Induction CTH + CCRT & 54 & $34.1 \%$ \\
Best supportive care & 9 & $5.7 \%$ \\
Total & 158 & $100 \%$ \\
\hline
\end{tabular}

Table 3. Radiotherapy course completion.

\begin{tabular}{cccc}
\hline Course completion & Yes & No & Total \\
\hline Radical Radiotherapy & $36(87.8 \%)$ & $5(12.2 \%)$ & $41(100 \%)$ \\
CCRT & $47(87 \%)$ & $7(13 \%)$ & $54^{*}(100 \%)$ \\
Induction CTH + CCRT & $42(77.8 \%)$ & $12(22.2 \%)$ & $54(100 \%)$ \\
Total & $125(3.9 \%)$ & $24(16.1 \%)$ & $149(100 \%)$ \\
\hline
\end{tabular}

*Including 2 patients who received Adjuvant CTH after the CCRT.

Eighty four percent of total number of included patients continued there radiotherapy course, course discontinuation was attributed to acute side effects in $37.5 \%$ of patients, socioeconomic factors in $12.5 \%$ of patients.

\subsection{Acute Side Effects}

Acute side effects of radiotherapy were properly reported for 104 patients. Mucositis was by far the most frequently encountered acute side effect in $98.1 \%$ of cases, followed by xerostomia in $93.3 \%$ of cases with grade 2 being the most frequent.

Patients who received radiotherapy by 2D technique exhibited higher incidence of acute toxicities compared to their counterparts who received 3DCRT, this was highly statistically significant for xerostomia with a p-value $>0.001$.

\subsection{Late Side Effects and Quality of Life}

The most frequently encountered late side effects were xerostomia \& dysphagia in $80 \%$ \& $44 \%$ of cases respectively.

The incidence of xerostomia, dysphagia, visual affection, hearing affection \& neck fibrosis was higher in patients treated with the 2D technique, with xerostomia being significantly lower in patients treated with 3D CRT.

\section{Treatment Outcomes}

\subsection{Local Control}

The local response was evaluated in 125 patients who completed the radiotherapy course; evaluation was done 2 - 4 months after treatment by either local imaging or nasopharyngeal endoscopy or both (Table 4).

The local control among all 149 patients was $48.3 \%$ at 3 years and $38.2 \%$ at 5 years (Figure 1 ).

Among all prognostic factors studied, only T-stage (T1-2 vs T3-4), RTH alone vs. RTH + CTH, and RTH course completion had statistically significant difference on both univariate \& multivariate analyses.

\subsection{Disease Free Survival (DFS)}

The DFS among all 149 patients was $42 \%$ at 3 years and 33.5\% at 5 years. 
Table 4. Factors affecting the 3-year \& 5-year local control.

\begin{tabular}{ccc}
\hline & Local response to treatment & \\
\hline Local response & Number of patients & Total \\
\hline Complete response (CR) & 50 & $40 \%$ \\
Partial response (PR) & 46 & $36.8 \%$ \\
Progressive disease (PD) & 10 & $8 \%$ \\
Not assessed & 19 & $15.2 \%$ \\
Total & 125 & $100 \%$ \\
\hline
\end{tabular}

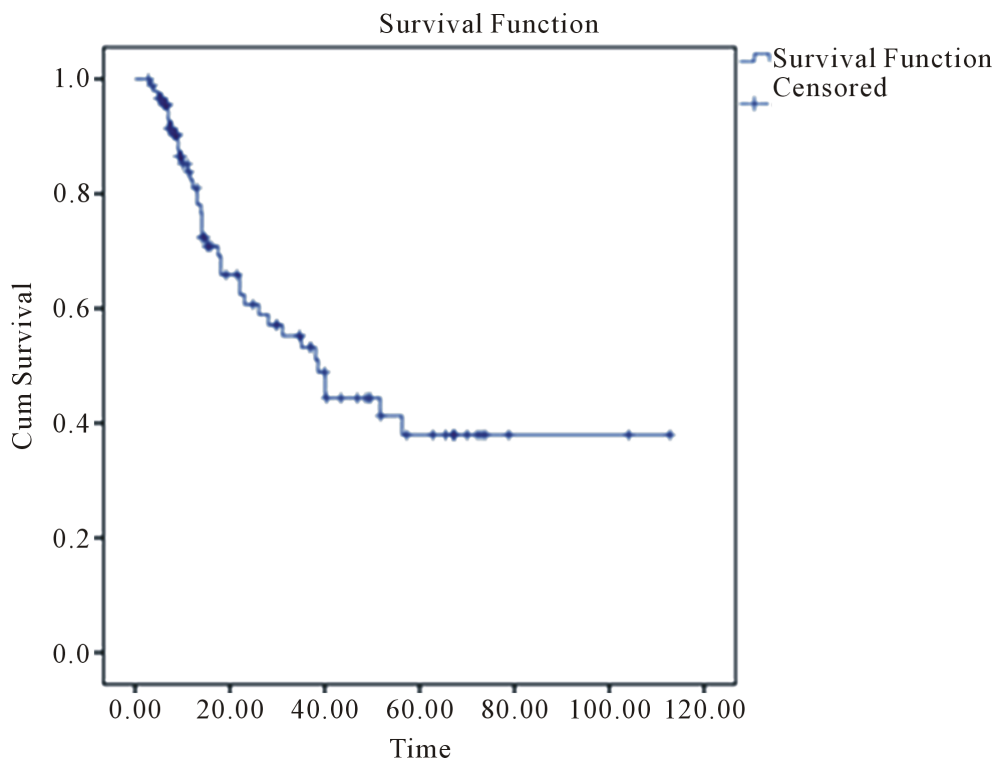

Figure 1. LC for non-metastatic NPC patients.

The performance status, T-Stage, the addition of chemotherapy, patients who succeeded to achieve a complete remission and radiotherapy course completion significantly influenced the DFS rates. Multivariate analysis showed them as independent indicators of DFS (Figure 2).

\subsection{Overall Survival (OS)}

The OS among all 158 patients was 50.9\% at 3 years and 37.2\% at 5 years (Figure 3).

Patients who were 50 years of age or younger, with performance status I, T1-2 tumor, non-metastatic disease, with stage groups I-III, and those who received chemotherapy in addition to radiotherapy, and those who completed radiotherapy course, and patients who succeeded to achieve a complete remission, had more favorable 3-year \& 5-year OS \& achieved significantly higher 3-year and 5-year OS rates on univariate analysis .on multivariate analysis performance status and stage group were not persistently significant.

\section{Discussion}

In this retrospective study, 158 patients with nasopharyngeal carcinoma presenting to the radiotherapy department at NCI Cairo from the year 2000 to 2010 were reviewed.

While nasopharyngeal carcinoma can occur in any age, Ferlay et al. 2010 [1] reported a bimodal age distribution, the first peak incidence arises between 15 to 25 years of age, with the second peak at 50 to 59 years of age. This was affirmed in our study, where the highest incidence was observed in the fifth (26.6\%), \& second (13.3\%) decades respectively. 


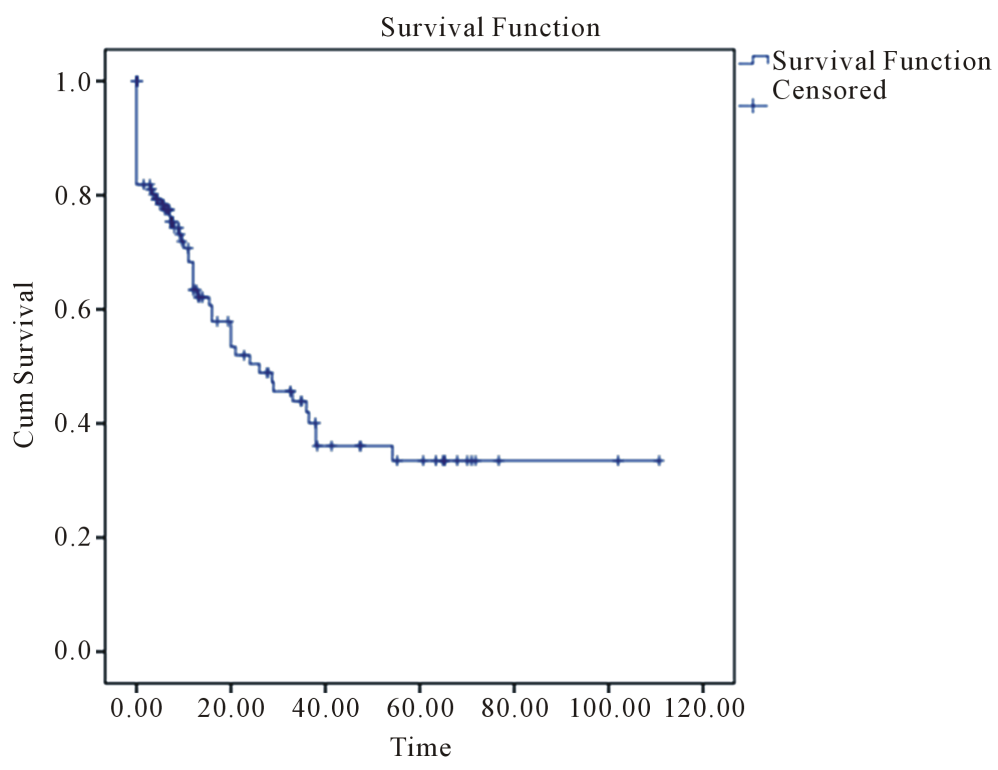

Figure 2. DFS for all non-metastatic NPC patients.

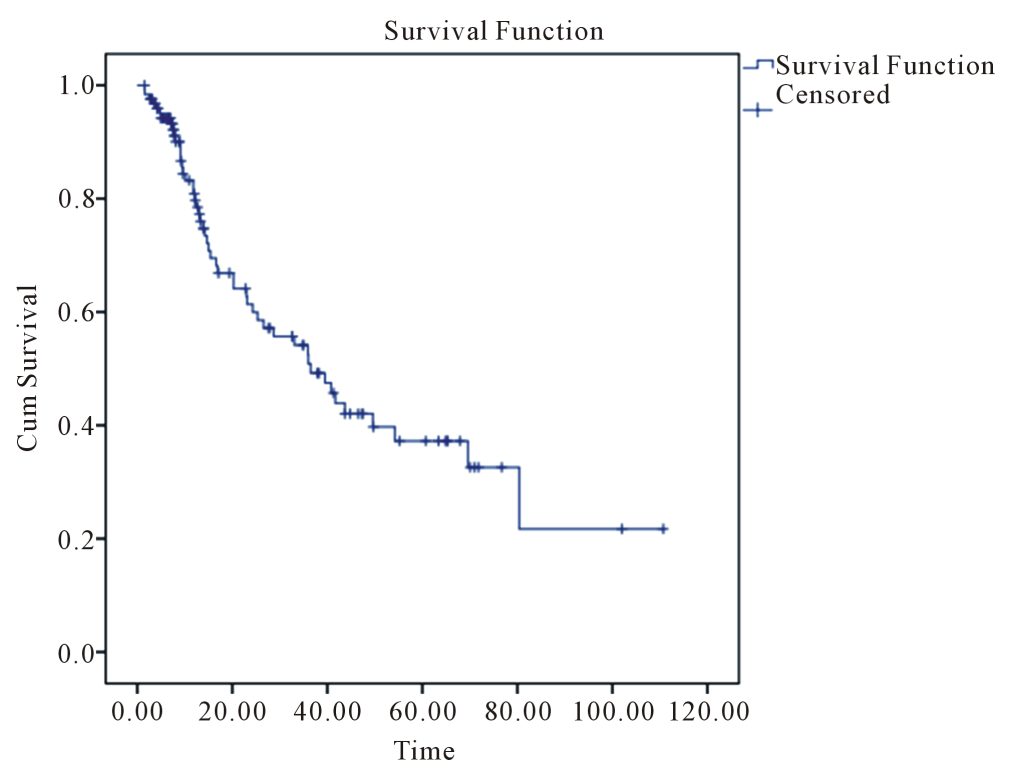

Figure 3. OS for all NPC patients.

The incidence of NPC is higher in males than that in females, and the ratio is $2-3: 1$ [1]. Similarly, in our study, male patients represented about $70 \%$ of patients.

Most of the patients in our study presented with WHO type III which constituted about $76.6 \%$ of all histological type. This came close to the study by Chee et al. (2013) [11] in which about $70.5 \%$ of the patient presented with WHO type III.

The vast majority of patients in our study presented at an advanced stage, as $86 \%$ of patients were diagnosed at stage III \& IV. Recently, a study from Malaysia analyzing the five-year survival of 134 NPC patients, reported similar findings, where $80 \%$ of patients presented at stage III \& IV [12], which was also supported by Chee et al. (2013) [11].

With regards to clinical presentation, it was found that $71 \%$ of cases presented with a neck swelling. This was entirely consistent with what was reported by Skinner et al. 1991 [13], and T. Dhaneshor et al., 2011 [14].

Acute toxicities were recorded during radiotherapy sessions. In the current study, we found that mucositis \& 
xerostomia are the most frequently encountered acute side effects, occurring in $98 \%$ \& 93.3\% respectively. This was nearly similar to what was reported by Lee et al. 2002 [15]. As regards the late toxicities, xerostomia \& dysphagia were the commonest late side effects. There was a significant reduction in xerostomia in patients who received 3D conformal radiotherapy. Similarly, in a study comparing the quality of life for NPC patients treated with different RT technologies, it was found that there was a significant difference in the incidence of xerostomia between patients treated using the 2D technique \& those receiving 3DCRT or IMRT [16].

Most of the studies analyzing the prognostic impact of age in NPC indicate that younger patients have a higher overall survival [17]-[20]. Some studies also showed that disease-related survival, local control and distant metastasis were affected by age [21]. In the current study, the overall survival rate in patients older than 50 years of age was significantly lower than in patients younger than 50 years of age $(p=0.001)$ by both univariate \& multivariate analyses, however, there was no significant difference in DFS between the two age groups ( $\mathrm{p}=$ 0.105), This was consistent with what was recently reported by Dou et al. 2014 [20].

Previous studies have reported slightly better, though not significant, long-term survival rates in women with NPC than in men [5] [22]. The same as we had reported in our study.

Xiao et al. 2013 [23] Found that male patients were more likely to have distant metastases than female patients and exhibited inferior overall survival and disease progression-free survival rates.

In a study, retrospectively, reviewed clinical outcome of 905 NPC patients treated by radiation alone during 1990 to 1999 from Mainland China, Karnofsky performance status showed no significant effect on overall and disease-free survival by multivariate analysis [24]. In the current study patients with ECOG PS I achieved significantly higher DFS \& OS by univariate analysis and on DFS only on multivariate analysis. This may be attributed to the fact that many of the patients with poor general condition received radiotherapy only despite being locally advanced as chemotherapy couldn't be tolerated.

Many studies have confirmed a clear association between long-term survival and NPC clinical stage [22] [25] [26]. Results of the current study also demonstrated that, as the disease stage increased, the 5-year OS rates significantly decreased $(\mathrm{p}=0.01)$ on univariate analysis, however the clinical stage didn't prove to be an independent prognostic factor of OS on multivariate analysis. Further analysis demonstrated that $\mathrm{T}$ stage and distant metastases (M) are decisive independent factors for OS, the T stage also significantly influenced the local control \& DFS on both univariate \& multivariate analyses. Similarly, Dou et al. 2014 reported that T-stage, \& M-stage are independent predictors of OS [20].

The efficacy of primary treatment was also found to be an independent prognostic factor affecting long-term survival by univariate \& multivariate analyses. The 5-year OS and DFS rates were significantly higher in patients with CR than those with PR ( $\mathrm{p}$ 0.001). This was consistent with what was reported by Dou et al. 2014 that the initial treatment modality and its therapeutic efficacy are the major factors affecting the prognosis of patients in all stages of NPC [20].

Many studies have shown that adding chemotherapy to radiotherapy can improve treatment efficacy and prolong OS in patients with intermediate or advanced stage NPC [27]-[30]. Two large meta-analysis studies showed superiority of concurrent chemo-radiotherapy (CCRT) compared to radio-therapy alone [31] [32].

In a meta-analysis by Langendijk et al. 2004, it was shown that the most efficacious way to introduce chemotherapy was concurrently with radiotherapy and this approach resulted in an absolute 5-year survival benefit of $20 \%$ [33]. Similarly, in the current study, patients who received chemotherapy in addition to radiotherapy achieved significantly higher local control, DFS, \& OS rates by both univariate \& multivariate analyses.

Several randomized controlled trials compared induction chemotherapy followed by concurrent chemoradiation versus chemoradiation with or without adjuvant chemotherapy in advanced NPC; [34]-[39], but none of them were large enough to show a statistically significant effect. Recently, Zhong-Guo et al. 2013 [40] conducted a meta-analysis of 1096 patients from 11 randomized controlled trials comparing efficacy of induction chemotherapy followed by concurrent chemoradiotherapy versus concurrent chemoradiotherapy with or without adjuvant chemotherapy. The study concluded that induction chemotherapy followed by concurrent chemo-radiotherapy couldn't improve OS, LFFS, and DMFS significantly. The authors recommended that larger and multicenteric randomized controlled trials are required to assess whether induction chemotherapy followed by CCRT is superior to CCRT with or without adjuvant chemotherapy for locoregionally advanced NPC. Similarly, in the current study induction chemotherapy didn't produce any statistically significant survival benefit.

In the treatment of nasopharyngeal carcinoma with radiotherapy, completed planned prescription dose and regular radiotherapy are vital [41]. In a recent study by Fei et al. 2013 [42], it was found that completion of the 
prescribed dose and radiotherapy regularity are related to the prognosis of nasopharyngeal carcinoma, multivariate analysis results revealed that treatment course completion and radiotherapy regularity are independent risk factors influencing survival with $p$ values $(p=0.003)$ and $(p=0.002)$ respectively. In the current study, we concluded that radiotherapy course completion is an independent prognostic factor influencing local control, as well as DFS \& OS rates.

The LC as well as the DFS \& OS rates in this study were very poor. This may be attributed to a number of reasons. First, over $86 \%$ of patients were locally advanced. Second, the $2 \mathrm{D}$ technique was used for the vast majority of patients in this study, which may have led to higher acute toxicities that would result in treatment interruptions \& radiotherapy course discontinuation [43].

\section{Conclusion and Recommendations}

This study matches the published data that support that radical concurrent chemoradiation is the mainstay of treatment of locally advanced NPC, \& that T-stage, M-stage, prescribed dose completion, response to initial treatment are independent prognostic factors for survival.

All measures should be taken to improve the local response during primary treatment as this will improve survival rates of patients with NPC.

Large and multicenteric randomized controlled trials are required to assess whether induction chemotherapy followed by concurrent chemoradiotherapy is superior to chemoradiotherapy for locoregionally advanced NPC.

New techniques like IMRT should be the standard of care for treatment of NPC.

\section{References}

[1] Ferlay, J., Shin, H., Bray, F., et al. (2010) GLOBOCAN 2008, Cancer Incidence and Mortality Worldwide: IARC Cancer Base No. 10. International Agency for Research on Cancer, Lyon.

[2] Chang, E.T. and Adami, H.-O. (2006) The Enigmatic Epidemiology of Nasopharyngeal Carcinoma. Cancer Epidemiology, Biomarkers \& Prevention, 15, 1765-1777. http://dx.doi.org/10.1158/1055-9965.EPI-06-0353

[3] Cao, S.M., Simons, M.J. and Qian, C.N. (2011) The Prevalence and Prevention of Nasopharyngeal Carcinoma in China. Chinese Journal of Cancer, 30, 114-119. http://dx.doi.org/10.5732/cjc.010.10377

[4] Edge, S.B. and Compton, C.C. (2010) American Joint Committee on Cancer: The 7th Edition of the AJCC Cancer Staging Manual and the Future of TNM. Annals of Surgical Oncology, 17, 1471-1474

[5] Wang, R.Z., Tan, Y., Wang, X.Y., Ma, L.L., Wang, D.M., Hu, Y.H. and Qin, Y.H. (2014) Prognoses and Long-Term Outcomes of Nasopharyngeal Carcinoma in Han and Uyghur Patients Treated with Intensity-Modulated Radiotherapy in the Xinjiang Autonomous Region of China. PLoS ONE, 9, e111145. http://dx.doi.org/10.1371/journal.pone.0111145

[6] Zhang, L., Chen, Q.Y., Liu, H., et al. (2013) Emerging Treatment Options for Nasopharyngeal Carcinoma. Drug Design, Development and Therapy, 7, 37-52.

[7] Spratt, D.E. and Lee, N. (2012) Current and Emerging Treatment Options for Nasopharyngeal Carcinoma. OncoTargets and Therapy, 5, 297-308.

[8] Zheng, Y., Han, F., Xiao, W., et al. (2015) Analysis of Late Toxicity in Nasopharyngeal Carcinoma Patients Treated with Intensity Modulated Radiation Therapy. Radiation Oncology, 10, 17. http://dx.doi.org/10.1186/s13014-014-0326-z

[9] Dawson, B. and Trapp, R.G., Eds. (2004) Basic and Clinical Biostatistics. 4th Edition, McGraw-Hill Professional.

[10] Oken, M.M., Creech, R.H., Tormey, D.C., et al. (1982) Toxicity and Response Criteria of the Eastern Cooperative Oncology Group. American Journal of Clinical Oncology, 5, 649-655. http://dx.doi.org/10.1097/00000421-198212000-00014

[11] Ee Phua, V.C., Loo, W.H., Yusof, M.M., Ishak, W.Z.W., Tho, L.M. and Ung, N.M. (2013) Treatment Outcome for Nasopharyngeal Carcinoma in University Malaya Medical Centre from 2004-2008. Asian Pacific Journal of Cancer Prevention, 14, 4567-4570. http://dx.doi.org/10.7314/APJCP.2013.14.8.4567

[12] Siti-Azrin, A.H., Norsa’adah, B. and Naing, N.N. (2014) Five-Year Survival and Median Survival Time of Nasopharyngeal Carcinoma in Hospital Universiti Sains Malaysia. Asian Pacific Journal of Cancer Prevention, 15, 6455-6459. http://dx.doi.org/10.7314/APJCP.2014.15.15.6455

[13] Skinner, D.W., Van Hasselt, C.A. and Tsao, S.Y. (1991) Nasopharyngeal Carcinoma: Modes of Presentation. Annals of Otology, Rhinology \& Laryngology, 100, 544-551. http://dx.doi.org/10.1177/000348949110000705

[14] Sharma, T.D., Singh, T.T., Laishram, R.S., Sharma, L.D., Sunita, A.K. and Imchen, L.T. (2011) Nasopharyngeal Car- 
cinoma-A Clinico-Pathological Study in a Regional Cancer Centre of Northeastern India. Asian Pacific Journal of Cancer Prevention, 12, 1583-1587.

[15] Lee, N., Xia, P., Quivey, J.M., Sultanem, K., Poon, I., Akazawa, C., et al. (2002) Intensity-Modulated Radiotherapy in the Treatment of Nasopharyngeal Carcinoma: An Update of the UCSF Experience. International Journal of Radiation Oncology, Biology, Physics, 53, 12-22. http://dx.doi.org/10.1016/S0360-3016(02)02724-4

[16] Jang-Chun, L., Jing-Min, H. and Yee-Min, J., et al. (2014) Comparisons of Quality of Life for Patients with Nasopharyngeal Carcinoma after Treatment with Different RT Technologies. ACTA Otorhinolaryngologica Italic, 34, 241-246.

[17] Lee, A.W., Sze, W.M., Au, J.S., Leung, S.F., Leung, T.W., Chua, D.T.T., et al. (2005) Treatment Results from Nasopharyngeal Carcinoma in the Modern Era: The Hong Kong Experience. International Journal of Radiation Oncology, Biology, Physics, 61, 1107-1116. http://dx.doi.org/10.1016/j.ijrobp.2004.07.702

[18] Yeh, S.A., Tang, Y., Lui, C.C., Huang, Y.-J. and Huang, E.-Y. (2005) Treatment Outcomes and Late Complications of 849 Patients with Nasopharyngeal Carcinoma Treated with Radiotherapy Alone. International Journal of Radiation Oncology, Biology, Physics, 62, 672-679. http://dx.doi.org/10.1016/j.ijrobp.2004.11.002

[19] Sze, H.C., Ng, W.T., Chan, O.S., Shum, T.C.Y., Chan, L.L.K. and Lee, A.W.M. (2012) Radical Radiotherapy for Nasopharyngeal Carcinoma in Elderly Patients: The Importance of Co-Morbidity Assessment. Oral Oncology, 48, 162-167. http://dx.doi.org/10.1016/j.oraloncology.2011.08.019

[20] Dou, H.Q., Hu, D.Y., Lam, C., Liu, Y.S., Wang, X.W. and Zhang, W.D. (2014) Retrospective Analysis of Results of Treatment for Nasopharyngeal Carcinoma in Macao. Chinese Journal of Cancer Research, 26, 148-158.

[21] Lai, S.Z., Li, W.F., Chen, L., Luo, W., Chen, Y.-Y., Liu, L.-Z., et al. (2011) How Does Intensity-Modulated Radiotherapy versus Conventional Two-Dimensional Radiotherapy Influence the Treatment Results in Nasopharyngeal Carcinoma Patients? International Journal of Radiation Oncology, Biology, Physics, 80, 661-668. http://dx.doi.org/10.1016/j.ijrobp.2010.03.024

[22] Phua, C.E., Tan, B.S., Yong, T.K. and Govindasamy, M. (2011) Retrospective Analysis of Results of Treatment for Nasopharyngeal Carcinoma in Penang General Hospital from 2001-2005. Asian Pacific Journal of Cancer Prevention, 12, 3197-3200.

[23] Xiao, G.L., Cao, Y.B., Qiu, X.B., Wang, W.H. and Wang, Y.F. (2013) Influence of Gender and Age on the Survival of Patients with Nasopharyngeal Carcinoma. BMC Cancer, 13, 226. http://dx.doi.org/10.1186/1471-2407-13-226

[24] Yi, J.L., Gao, L., Huang, X.D., Li, S.-Y., Luo, J.-W., Cai, W.-M., et al. (2006) Nasopharyngeal Carcinoma Treated by Radical Radiotherapy Alone: Ten-Year Experience of a Single Institution. International Journal of Radiation Oncology, Biology, Physics, 65, 161-168. http://dx.doi.org/10.1016/j.ijrobp.2005.12.003

[25] Kawashima, M., Fuwa, N., Myojin, N., Nakamura, K., Toita, T., Saijo, S., et al. (2004) A Multi-Institutional Survey of the Effectiveness of Chemotherapy Combined with Radiotherapy for Patients with Nasopharyngeal Carcinoma. Japanese Journal of Clinical Oncology, 34, 569-583. http://dx.doi.org/10.1093/jjco/hyh111

[26] Goto, Y., Kodaira, T., Fuwa, N., Mizoguchi, N., Nakahara, R., Nomura, M., et al. (2013) Alternating Chemoradiotherapy in Patients with Nasopharyngeal Cancer: Prognostic Factors and Proposal for Individualization of Therapy. Journal of Radiation Research, 54, 98-107. http://dx.doi.org/10.1093/jrr/rrs071

[27] Chua, D.T., Ma, J., Sham, J.S., Mai, H.-Q., Choy, D.T.K., Hong, M.-H., et al. (2005) Long-Term Survival after Cisplatin-Based Induction Chemotherapy and Radiotherapy for Nasopharyngeal Carcinoma: A Pooled Data Analysis of Two Phase III Trials. Journal of Clinical Oncology, 23, 1118-1124. http://dx.doi.org/10.1200/JCO.2005.12.081

[28] Park, K.H., Kim, J.S., Park, Y., Seo, H.Y., Park, Y.J., Choi, I.K., et al. (2010) Concurrent Chemoradiation Followed by Adjuvant Chemotherapy in Patients with Locoregionally Advanced Nasopharyngeal Carcinoma in Korea. Cancer Chemotherapy and Pharmacology, 66, 643-651. http://dx.doi.org/10.1007/s00280-009-1203-6

[29] Lee, A.W., Tung, S.Y., Chua, D.T., Ngan, R.K.C., Chappell, R., Tung, R., et al. (2010) Randomized Trial of Radiotherapy plus Concurrent-Adjuvant Chemotherapy vs Radiotherapy Alone for Regionally Advanced Nasopharyngeal Carcinoma. Journal of the National Cancer Institute, 102, 1188-1198. http://dx.doi.org/10.1093/jnci/djq258

[30] Chen, Y., Sun, Y., Liang, S.B., Zong, J.-F., Li, W.-F., Chen, M., et al. (2013) Progress Report of a Randomized Trial Comparing Long-Term Survival and Late Toxicity of Concurrent Chemoradiotherapy with Adjuvant Chemotherapy versus Radiotherapy Alone in Patients with Stage III to IVB Nasopharyngeal Carcinoma from Endemic Regions of China. Cancer, 119, 2230-2238. http://dx.doi.org/10.1002/cncr.28049

[31] Yang, A.K., Liu, T.R., Guo, X., Qi, G.L., Chen, F.J., Guo, Z.M., et al. (2008) Concurrent Chemoradiotherapy versus Radiotherapy Alone for Locoregionally Advanced Nasopharyngeal Carcinoma: A Meta-Analysis. Chinese Journal of Otorhinolaryngology Head and Neck Surgery, 43, 218-223.

[32] Zhang, L., Zhao, C., Ghimire, B., Hong, M.-H., Liu, Q., Zhang, Y., et al. (2010) The Role of Concurrent Chemoradiotherapy in the Treatment of Locoregionally Advanced Naso-Pharyngeal Carcinoma among Endemic Population: A Meta-Analysis of the Phase III Randomized Trials. BMC Cancer, 10, 558. http://dx.doi.org/10.1186/1471-2407-10-558 
[33] Langendijk, J.A., Leemans, C.R., Buter, J., Berkhof, J. and Slotman, B.J. (2004) The Additional Value of Chemotherapy to Radiotherapy in Locally Advanced Nasopharyngeal Carcinoma: A Meta-Analysis of the Published Literature. Journal of Clinical Oncology, 22, 4604-4612. http://dx.doi.org/10.1200/JCO.2004.10.074

[34] He, A.J. (2011) Neoadjuvant Chemotherapy Followed by Concurrent Chemoradiotherapy in the Treatment of Locally Advanced Nasopharyngeal Carcinoma. Hebei Medical Journal, 33, 2115-2116.

[35] Xu, M.J., Ye, J.M., Wu, L.Q. and Wang, X.C. (2011) Study of TPF Neoadjuvant Chemotherapy Followed by Concurrent Chemoradiotherapy with Cisplatin in Locally Advanced Nasopharyngeal Carcinoma. Journal of Practical Oncology, 26, 291-294.

[36] Chen, X.J., Qiu, R.L., Yang, C.L. and Liu Y.Q. (2012) Study of TPF Neoadjuvant Chemotherapy Followed by Concurrent Intensity Modulated Radiochemotherapy in the Treatment for 30 Cases with Locally Advanced Nasopharyngeal Carcinoma. Chinese Journal of Clinical Oncology and Rehabilitation, 19, 201-204.

[37] Cui, J.D., Long, G.N., Zhang, Y., Li, T., Xu, Z.H., Wen, S.L. and Liang, C.L. (2012) Study of TPF Neoadjuvant Chemotherapy Followed by Concurrent Intensity Modulated Radiochemotherapy in the Treatment for 30 Cases with Locally Advanced Nasopharyngeal Carcinoma. Chinese Clinical Oncology, 17, 524-529.

[38] Fountzilas, G., Ciuleanu, E., Bobos, M., Kalogera-Fountzila, A., Eleftheraki, A.G., Karayannopoulou, G., et al. (2012) Induction Chemotherapy Followed by Concomitant Radiotherapy and Weekly Cisplatin versus the Same Concomitant Chemoradiotherapy in Patients with Nasopharyngeal Carcinoma: A Randomized Phase II Study Conducted by the Hellenic Cooperative Oncology Group (HeCOG) with Biomarker Evaluation. Annals of Oncology, 23, 427-435. http://dx.doi.org/10.1093/annonc/mdr116

[39] Huang, S., Deng, G., Huang, G., Li, Y., Meng, Y. and Chen, J. (2012) Efficacy of Induction Chemotherapy Combined with Concurrent Chemoradiotherapy for Advanced Nasopharyngeal Carcinoma. Chinese Journal of Clinical Oncology, 39, 788-791.

[40] Liang, Z.-G., Zhu, X.-D., Tan, A.-H., Jiang, Y.-M., Qu, S., Su, F. and Xu, G.-Z. (2013) Induction Chemotherapy Followed by Concurrent Chemoradiotherapy versus Concurrent Chemoradiotherapy with or without Adjuvant Chemotherapy for Locoregionally Advanced Nasopharyngeal Carcinoma: Meta-Analysis of 1,096 Patients from 11 Randomized Controlled Trials. Asian Pacific Journal of Cancer Prevention, 14, 515-521. http://dx.doi.org/10.7314/APJCP.2013.14.1.515

[41] O’Meara, W.P. and Lee, N. (2005) Advances in Nasopharyngeal Carcinoma. Current Opinion in Oncology, 17, 225230. http://dx.doi.org/10.1097/01.cco.0000156197.29872.8e

[42] Kong, F., Cai, B.-Z., Chen, X.-Z., Zhang, J. and Wang, Y.-M. (2013) Prognostic Factors for Survival of Patients with Nasopharyngeal Carcinoma Following Conventional Fractionation Radiotherapy. Experimental and Therapeutic Medicine, 6, 57-60. http://dx.doi.org/10.3892/etm.2013.1100

[43] Wu, S. and Zhao, C. (2000) The Impact of Radiotherapy Interruption and Time Delay on the Clinical Outcomes of Nasopharyngeal Carcinoma Patients. Chinese Journal of Cancer, 19, 923-926. 